\title{
INFLUENCE OF LAYER HEIGHT, BUILD ORIENTATION AND POST Curing on Tensile Mechanical Properties OF SLA 3D PRINTED MATERIAL
}

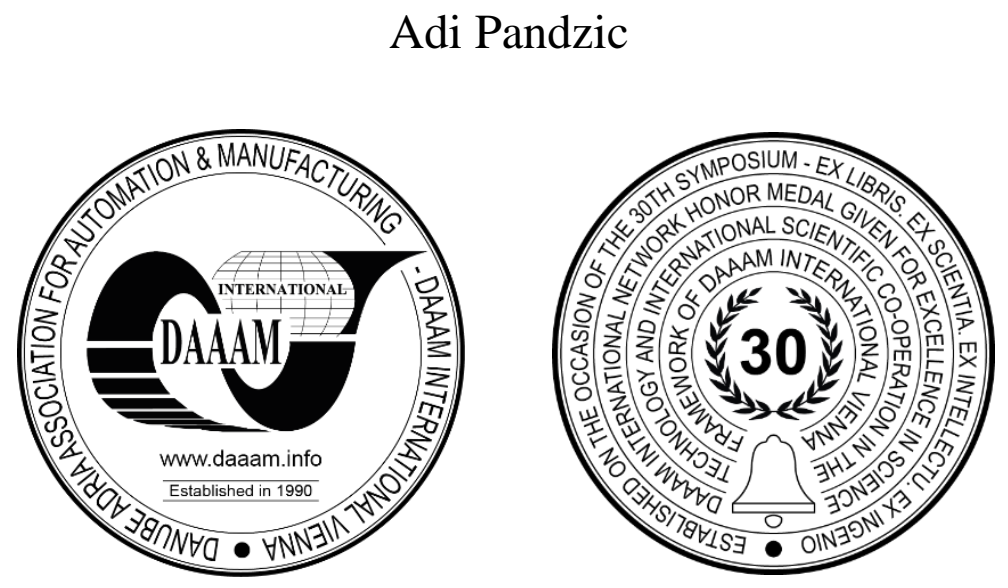

This Publication has to be referred as: Pandzic, A[di] (2021). Influence of Layer Height, Build Orientation and Post Curing on Tensile Mechanical Properties of SLA 3D Printed Material, Proceedings of the 32nd DAAAM International Symposium, pp.0200-0208, B. Katalinic (Ed.), Published by DAAAM International, ISBN 978-3-902734-33-4, ISSN 1726-9679, Vienna, Austria

DOI: $10.2507 / 32$ nd.daaam.proceedings.030

\begin{abstract}
In this research, an experimental analysis of the influence of layer height, build orientation and post curing on the tensile mechanical properties of SLA 3D printed material was performed. The material "Grey Resin" from the manufacturer Fromlabs was specifically analysed. When it comes to layer height, four different layer heights were analysed, with 160 , 100, 50, and 25 microns. Specimens were manufactured in three different build orientations (vertical, side and flat). All specimens were tested with and without post curing $\left(30 \mathrm{~min}, 60^{\circ} \mathrm{C}\right)$. The results in further text showed that the layer height, build orientation as well as the post curing have an impact on the tensile mechanical properties of the SLA "Grey Resin" material. Also, in addition to the effect on mechanical properties, the paper presents how these factors affect the time of 3D printing.
\end{abstract}

Keywords: SLA; Layer thickness; Build orientation; Curing; Tensile testing

\section{Introduction}

The technologies that are slowly changing the way products are made today are certainly additive technologies. Production of very complex physical models geometries with good quality and in a wide range of different materials is possible with additive manufacturing (AM) technologies. This technology is used in many fields such as engineering, medicine (prosthetics, bionics, orthotics), automotive and aircraft industries, dental restorations, prototyping, education and more [1], [2] [3]. Today it can be seen the continuous development of new AM technologies, materials and applications. In terminology additive manufacturing is also known as rapid prototyping (RP). On the market there are several different groups (families) of AM technologies (according to ASTM F2792 standard) such as VAT Photopolymerization, Powder Bed Fusion (PBF), Binder Jetting, Material Jetting, Sheet Lamination, Material Extrusion, Directed Energy Deposition (DED) and Hybrid, also all the listed technologies can be found in literature under different names. In this research study, stereolithography (SLA) AM technology was used, which in addition to DLP (Digital Light Processing), 3SP (Scan, Spin and Selectively Photocure) and CLIP (Continuous Liquid Interface Production) belongs to VAT Photopolymerization group (Fig. 1) [4], [5], [6]. 


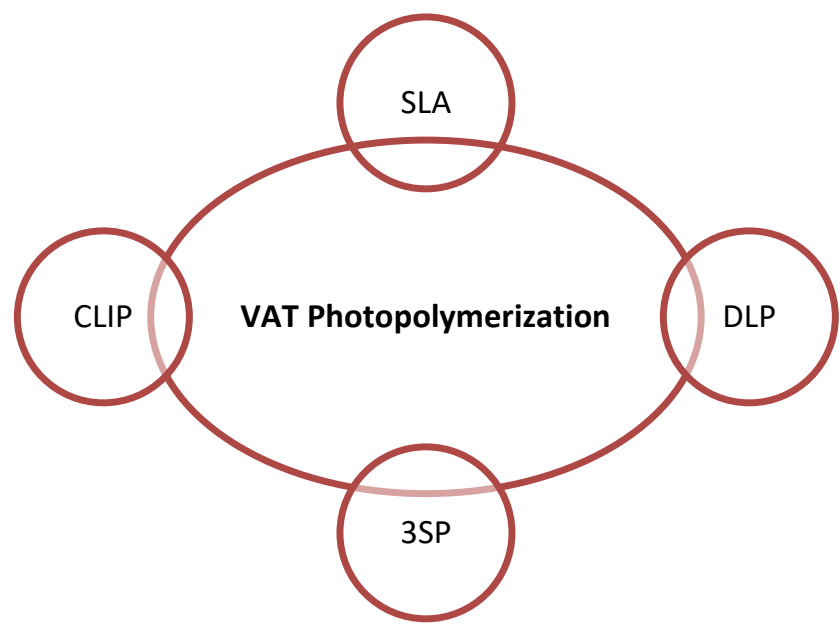

Fig. 1. VAT Photopolymerization AM “family" according to ASTM F2792 standard

With AM technologies, the first step is creating a 3D CAD model of the product using some of CAD software such as Solidworks, Catia, NX or others. The 3D CAD model is then converted to STL or another similar format, and using "Slicer" software, g-code with all printing parameters is generated which is further used by 3D printer to 3D print the product. In general, slicer cuts a 3D model into 2D cross-sectional layers, and based on that the 3D printer makes the final product layer by layer. [7], [8].

Stereolithography 3D printing technology is known for being able to produce products of simple but also complex geometries with high resolution (up to 25 microns) and accuracy, watertight and isotropic characteristics with smooth surface finish and fine features. SLA is technology that basically uses polymeric materials, known as resins, but today it can be seen that some companies developing also composites (Formlabs Ceramic Resin) [9], [10], [11]. There are a number of different materials (resins) on the market as well as the manufacturers themselves, and often the resins are divided by purpose as shown on Fig. 2.

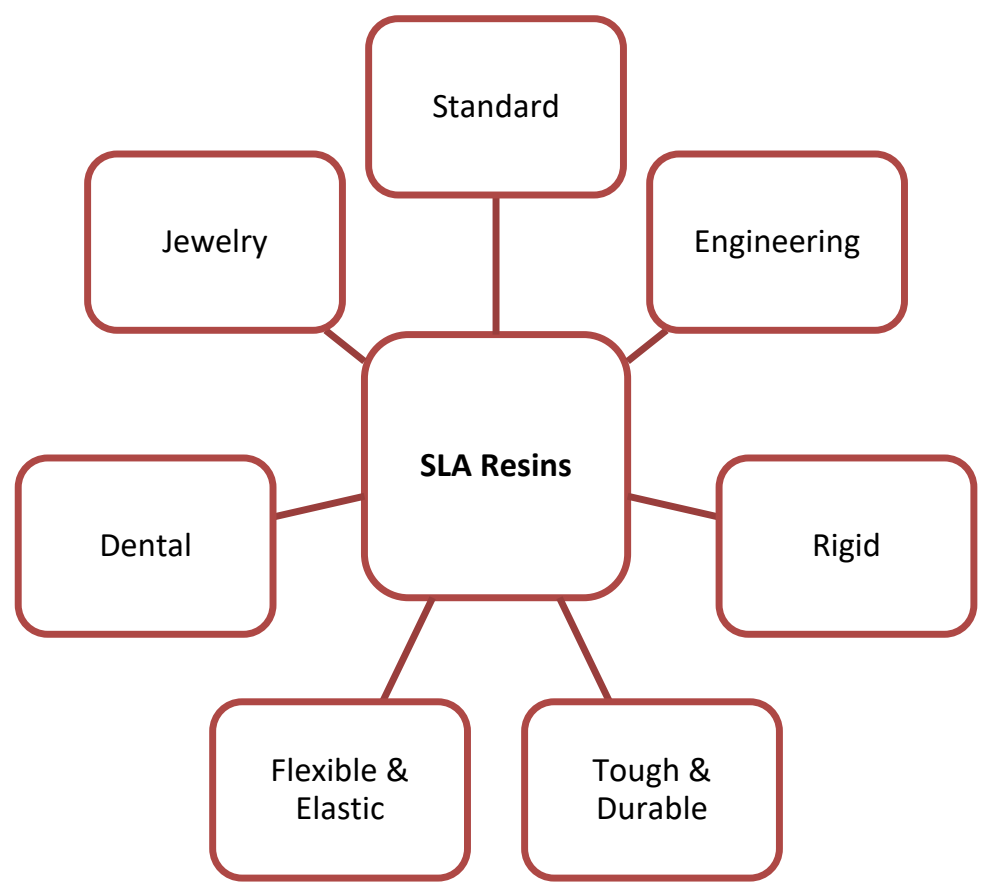

Fig. 2. SLA materials (resins) by Formlabs company

Working principle of SLA 3D printer is based on hardening (curing) a liquid photopolymer resin layer by layer using electronically controlled UV laser beam. The parts are 3D printed on a moving build platform located above fixed resin tank where the material (resin) is stored. Generally, final parts are created bottom-up, and when each new layer is made, they are immersed in the liquid resin. And this procedure is repeated until final part is completely 3D printed [12], [13]. Also, schematic representation of the basic mechanics and parts of SLA 3D printer (Formlabs Form 3) is presented on Fig. 3. 


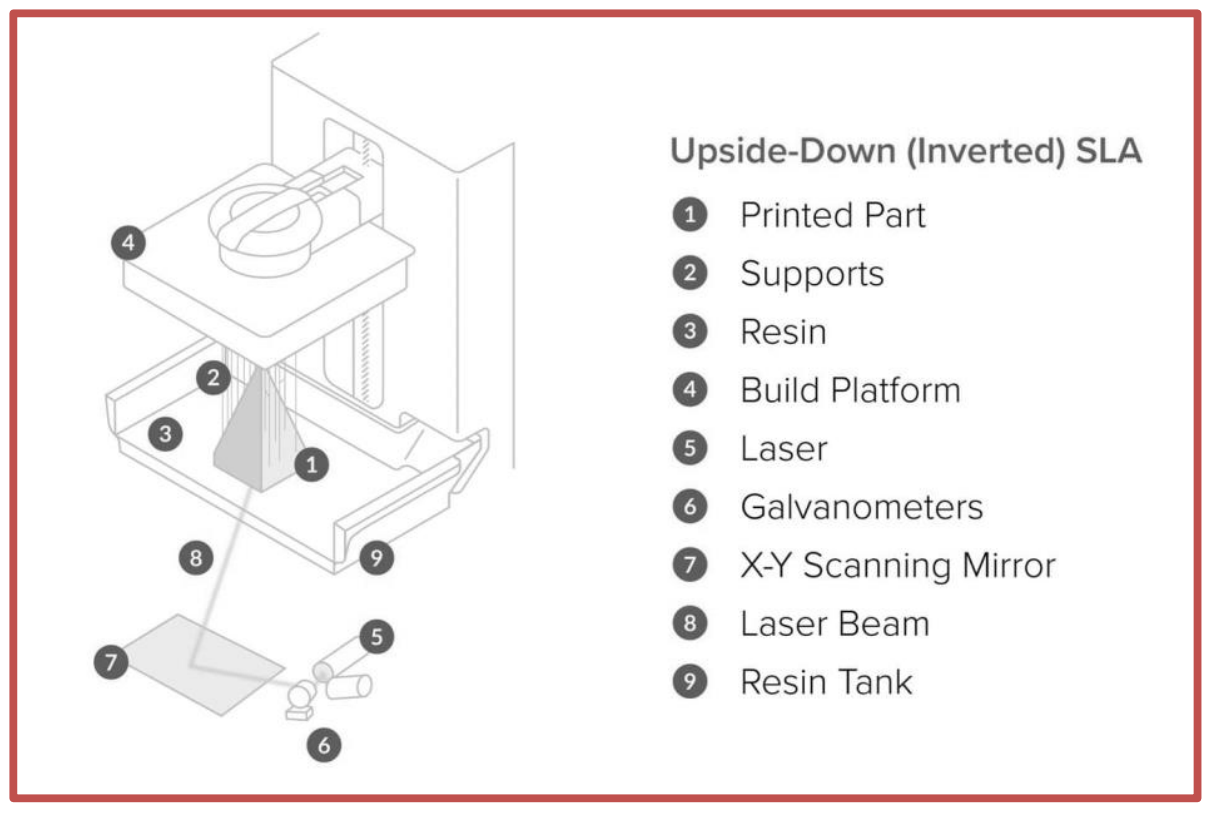

Fig. 3. Schematic representation of the basic mechanics of SLA 3D printer (figure taken from formlabs.com)

Once the final product is 3D printed and material solidifies, the build platform of the SLA printer is lifted out and the excess liquid drains away. After this, printed part usually goes in bath with IPA (isopropanol) alcohol to be washed. Washing can be manually with hands or automated using for example "Form Wash" where time can be controlled, and better cleaning can be achieved due to the circulation of IPA alcohol during the washing. After washing and drying printed product can be used or it can be additionally post cured, with different time and temperature which depends on type of material. Post curing helps 3D printed parts achieve their highest possible strength and stability [14], [15], [16].

Through a literature review, it can be concluded that mechanical properties of printed material in SLA technology are influenced by various factors such as: layer height, build orientation, supports, washing time, post curing time and temperature (Fig. 4). Also, if SLA technology is compared to FDM technology, here is a significant smaller number of influencing factors on the final characteristics of the printed product that user can change [17], [18], [19].

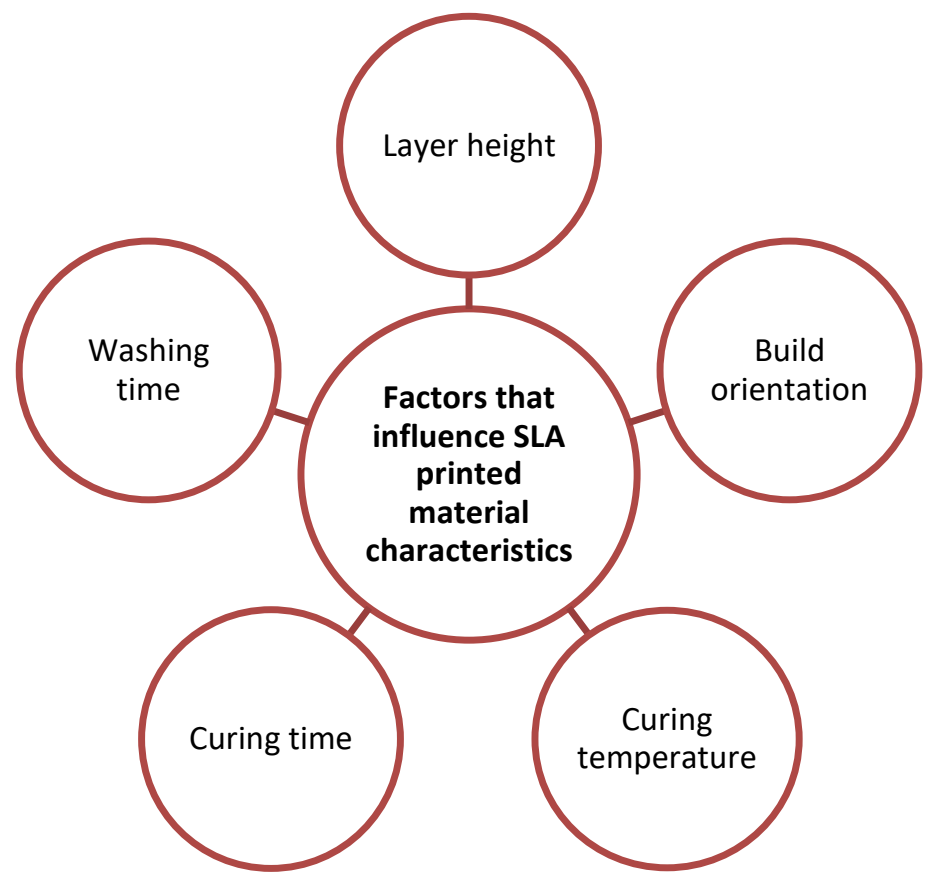

Fig. 4. Influencing factors and printing parameters on SLA printed material characteristics

Based on this, the idea of this paper is to analyse, through experimental research and statistical analysis of the obtained data, the influence of layer height, build orientation and post curing on the tensile mechanical properties of SLA printed material and the printing time. 


\section{Experimental details}

The experiment of this study is divided into several phases shown in the flow diagram below (Fig. 5). In the first stage, the CAD 3D model of tensile testing specimens has been designed and in STL format prepared. Than, in slicer software g-code with printing parameters was prepared and specimens were SLA 3D printed. All specimens are washed after 3D printing in IPA alcohol and half of the total amount of specimens were post cured. In the end all specimens were tensile tested, collected data was analysed and results are presented.

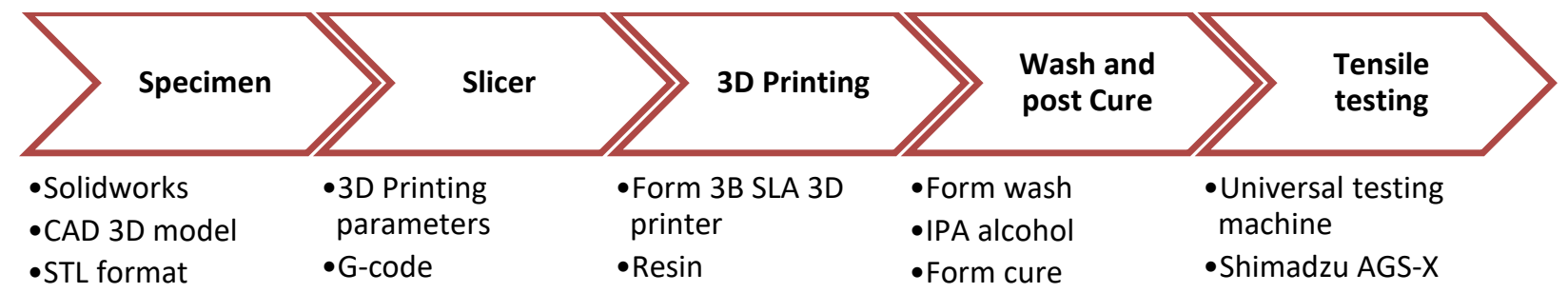

Fig. 5. Experiment phases flow diagram

CAD 3D model of tensile testing specimen was designed in Solidworks 2021 software, and STL format was prepared. Tensile testing specimen dimensions are according to ISO 527-2, and technical drawing is presented in Fig. 6.

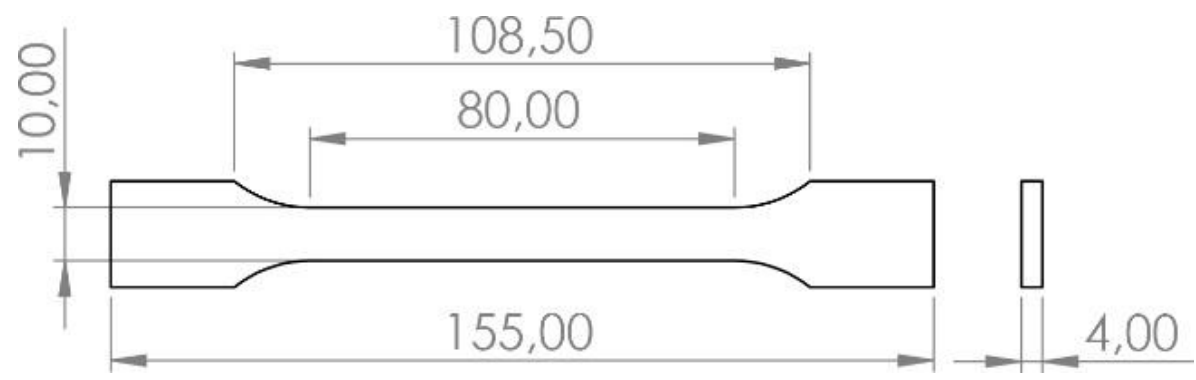

Fig. 6. Tensile testing specimen dimensions according to ISO 527-2

Specimen 3D model was prepared for printing in slicer software PreForm (FormLabs, 3.16.0 version), where printing parameters are also defined and presented in Table 1.

\begin{tabular}{|c|c|c|c|}
\hline 3D Printer & Resin (Material) & Layer height [microns] & Build orientation \\
\hline \multirow{3}{*}{ Form 3B } & \multirow{3}{*}{ Grey, V4 } & 25 & \multirow{2}{*}{ Vertical (V) } \\
\cline { 3 - 3 } & & 50 & Flat (F) \\
\cline { 3 - 3 } & & 100 & Side (S) \\
\cline { 3 - 3 } & & 160 & \\
\hline
\end{tabular}

Table 1. Printing parameters defined in PreForm slicer

Different build orientations are presented on Fig. 7. Also, the tensile test specimens in the PreForm slicer as well as after they were manufactured are shown in the Fig. 8.
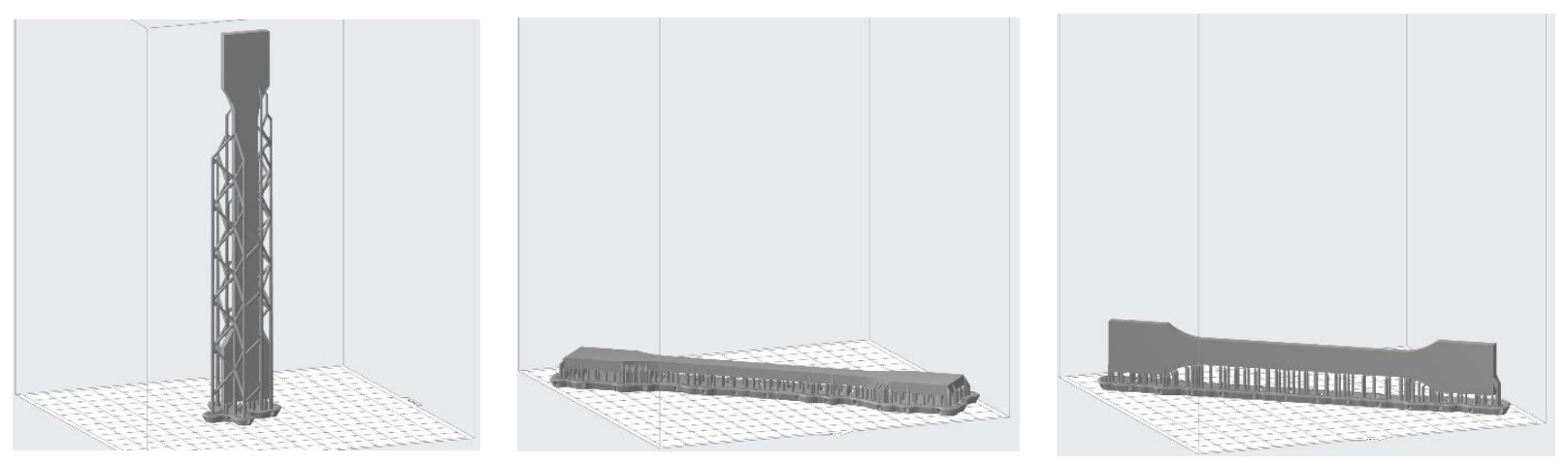

Fig. 7. Different specimen printing build orientations (from left: vertical, flat and side) 

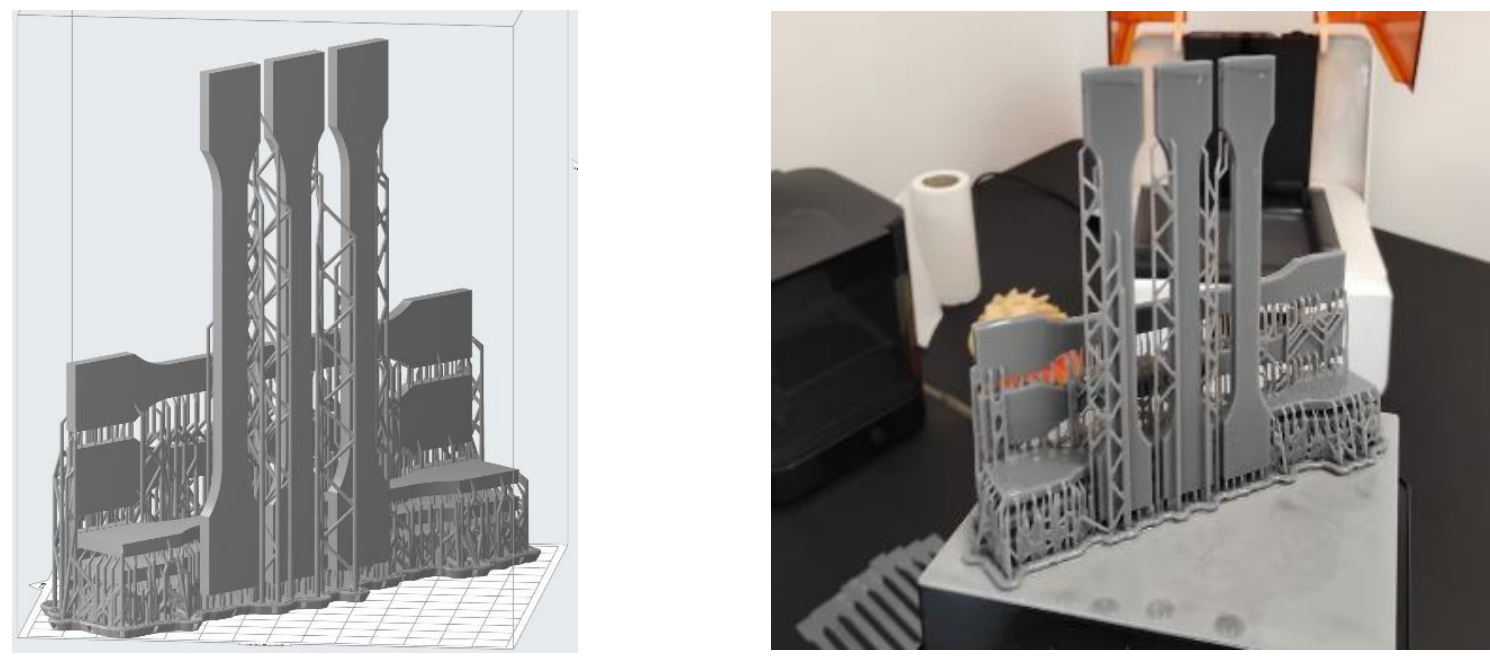

Fig. 8. Prepared tensile specimens in PreForm slicer (left) and printed specimens (right)

Grey resin (SLA material), from FormLabs manufacturer, was analysed in this paper. It is from group of standard resins for 3D printing strong, precise concept models and prototypes. With a matte surface finish, opaque appearance, and precise details, prints can be used right of the printer but also can be post cured. Material properties data by manufacturer is presented in Table 2.

\begin{tabular}{|c|c|c|c|}
\hline Material properties & Air dried without curing ${ }^{1}$ & Post-Cured ${ }^{2}$ & Method \\
\hline Ultimate tensile strength & $38 \mathrm{MPa}$ & $65 \mathrm{MPa}$ & ASTM D 638-10 \\
\hline Tensile modulus & $1,6 \mathrm{GPa}$ & $2,8 \mathrm{GPa}$ & ASTM D 638-10 \\
\hline Elongation at failure & $12 \%$ & $6,2 \%$ & ASTM D 638-10 \\
\hline Flexural modulus & $1,25 \mathrm{GPa}$ & $2,2 \mathrm{GPa}$ & ASTM D 790-10 \\
\hline Notched IZOD & $16 \mathrm{~J} / \mathrm{m}$ & $25 \mathrm{~J} / \mathrm{m}$ & ASTM D 256-10 \\
\hline \multicolumn{4}{|c|}{$\begin{array}{l}{ }^{1} \text { Data was obtained from parts, printed using Form } 2,100 \text { microns, Clear settings, washed and air dried without post } \\
\text { cure. } \\
{ }^{2} \text { Data was obtained from parts printed using Form 2, } 100 \text { microns, Clear setings, and post cured with } 1,25 \mathrm{~mW} / \mathrm{cm}^{2} \text { o } \\
405 \mathrm{~nm} \text { LED light for } 60 \text { minutes at } 60^{\circ} \mathrm{C} \text {. }\end{array}$} \\
\hline
\end{tabular}

Table 2. Grey resin material properties data by manufacturer

After setting all parameters in slicer, g-code was created, and all specimens were printed on Form 3B SLA 3D printer. Then, specimens were washed using Form Wash for $10 \mathrm{~min}$ and air dried. Post curing settings were 30 min with $60{ }^{\circ} \mathrm{C}$.

Tensile testing was performed according to ISO 527-2 and using universal testing machine Shimadzu AGS-X with maximum load of $10 \mathrm{kN}$. Testing speed (strain rate) was $5 \mathrm{~mm} / \mathrm{min}$, and all specimens were tested under laboratory conditions. Data monitoring during tensile testing was performed using Shimadzu Trapezium X software, and overall stress-strain diagrams for specimens with and without post curing are presented on Fig. 9.

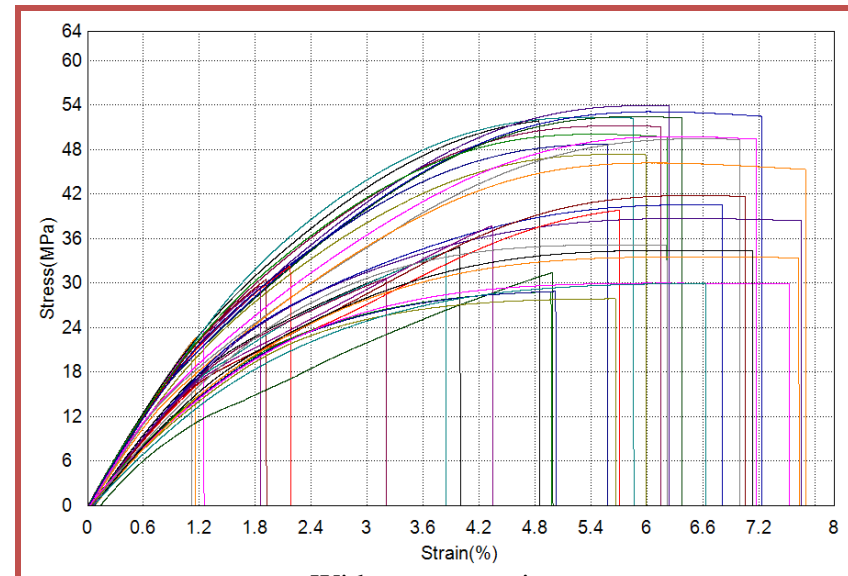

Without post curing

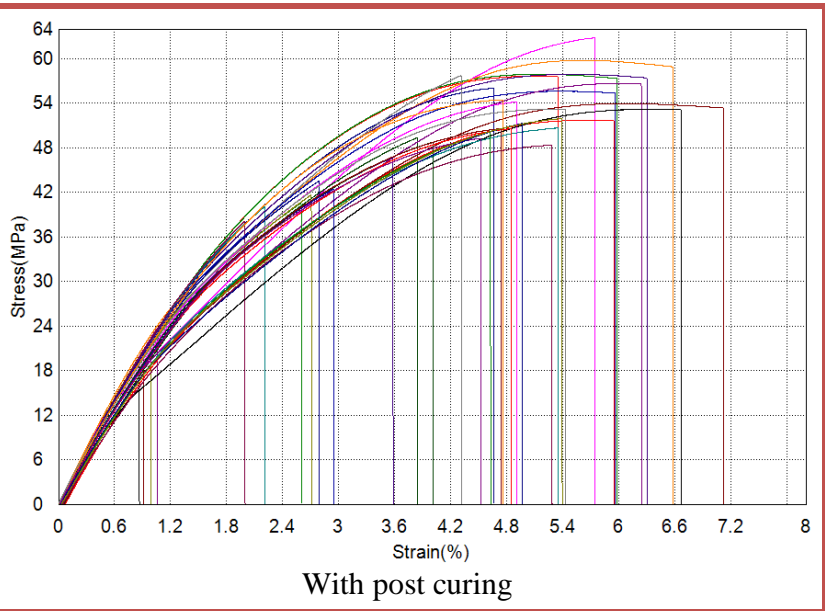

With post curing

Fig. 9. Overall Stress-Strain diagrams for tensile tested specimens with and without post curing 


\section{Results and discussion}

In this section tensile testing results are presented and discussed, where influence of layer height (LH), build orientation (BO) and post curing (PC) on tensile strength and elastic modulus were analysed. Also, influence of those parameters on printing time was analysed. In general, the results are presented through diagrams where they are analysed and commented.

Influence of layer height and post curing on tensile strength and elastic modulus for specimens printed in flat $(\mathrm{F})$ build orientation are presented on Fig. 10.
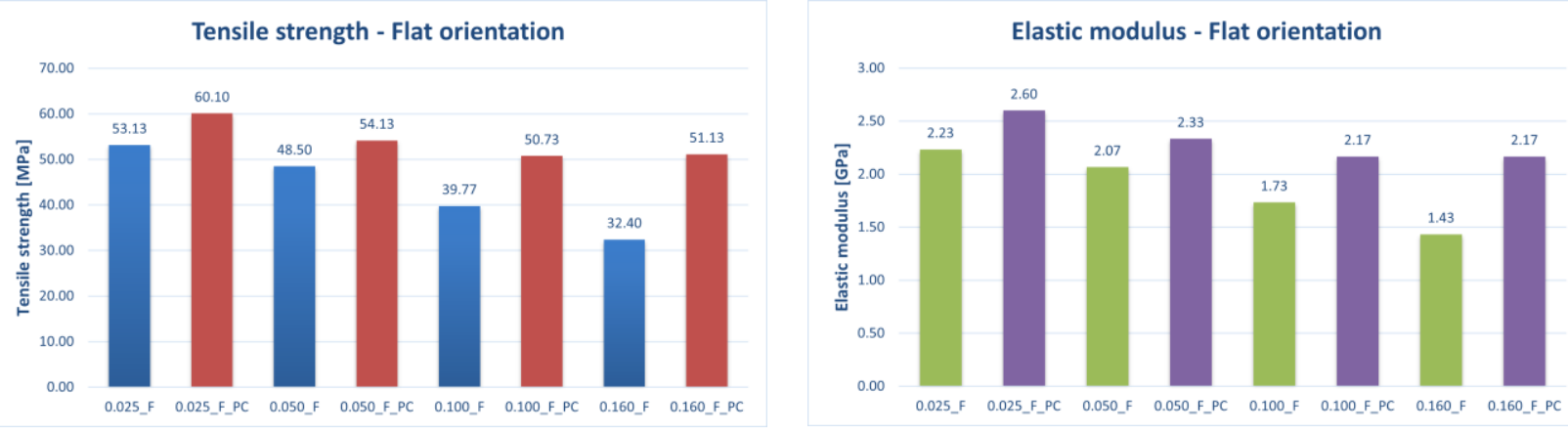

Fig. 10. Influence of $\mathrm{LH}$ and PC on tensile strength and elastic modulus for specimens printed in flat $\mathrm{BO}$

Diagrams from Fig. 10 are showing that LH have influence on tensile strength and elastic modulus for specimens printed in flat BO. Depending on LH tensile strength varies up to $48 \%$ for specimens without PC, and up to $17 \%$ for specimens with PC. The value of the elastic modulus varies up to $43 \%$ for specimens without PC, and up to $18 \%$ for specimens with PC. When analysing the PC effect, it can be seen that in general the value of the tensile strength and elastic modulus has increased. Depending on the LH, the PC caused an increase in tensile strength up to $44 \%$, and for elastic modulus up to $41 \%$.

Influence of layer height and post curing on tensile strength and elastic modulus for specimens printed in side (S) build orientation are presented on Fig. 11.
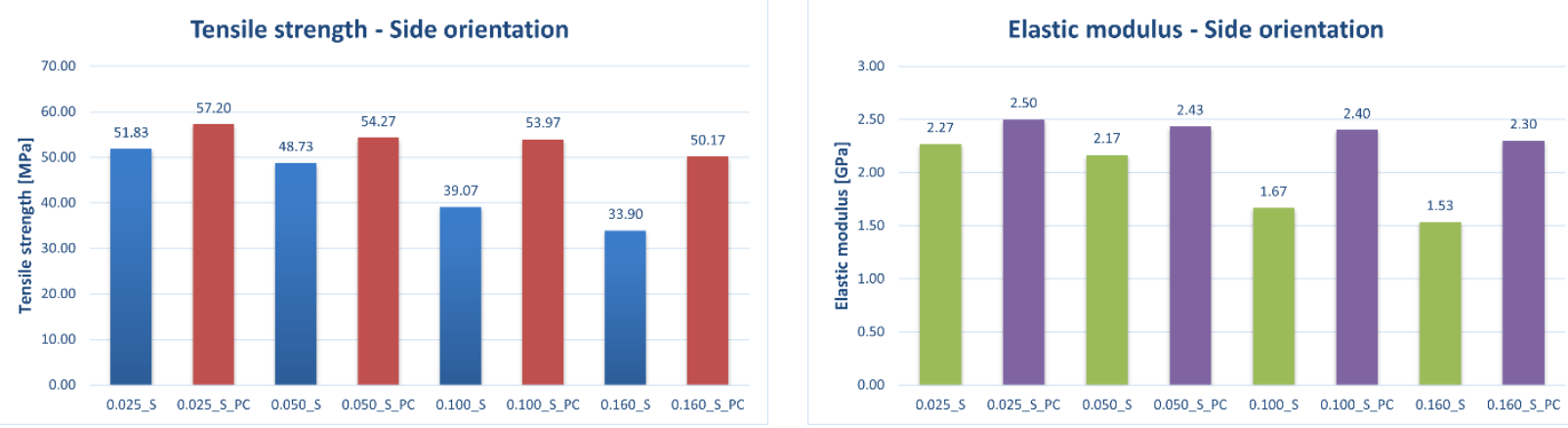

Fig. 11. Influence of LH and PC on tensile strength and elastic modulus for specimens printed in side BO

Diagrams from Fig. 11 are showing that LH have influence on tensile strength and elastic modulus for specimens printed in side BO. Depending on LH tensile strength varies up to $41 \%$ for specimens without PC, and up to $13 \%$ for specimens with PC. The value of the elastic modulus varies up to $39 \%$ for specimens without PC, and up to $8 \%$ for specimens with PC. When analysing the PC effect, it can be seen that the value of the tensile strength and elastic modulus has increased. Depending on the LH, the PC caused an increase in tensile strength up to $38 \%$, and for elastic modulus up to $40 \%$.

Influence of layer height and post curing on tensile strength and elastic modulus for specimens printed in vertical (S) build orientation are presented on Fig. 12, and diagrams are showing that LH have influence on tensile strength and elastic modulus for specimens printed in vertical BO. Depending on LH tensile strength varies up to $37 \%$ for specimens without PC, and up to $71 \%$ for specimens with PC. The value of the elastic modulus varies up to $46 \%$ for specimens without PC, and up to $7 \%$ for specimens with PC. When analysing the PC effect, in general the value of the tensile strength and elastic modulus has increased. Depending on the LH, the PC caused an increase in tensile strength up to $48 \%$, and for elastic modulus up to $47 \%$. 

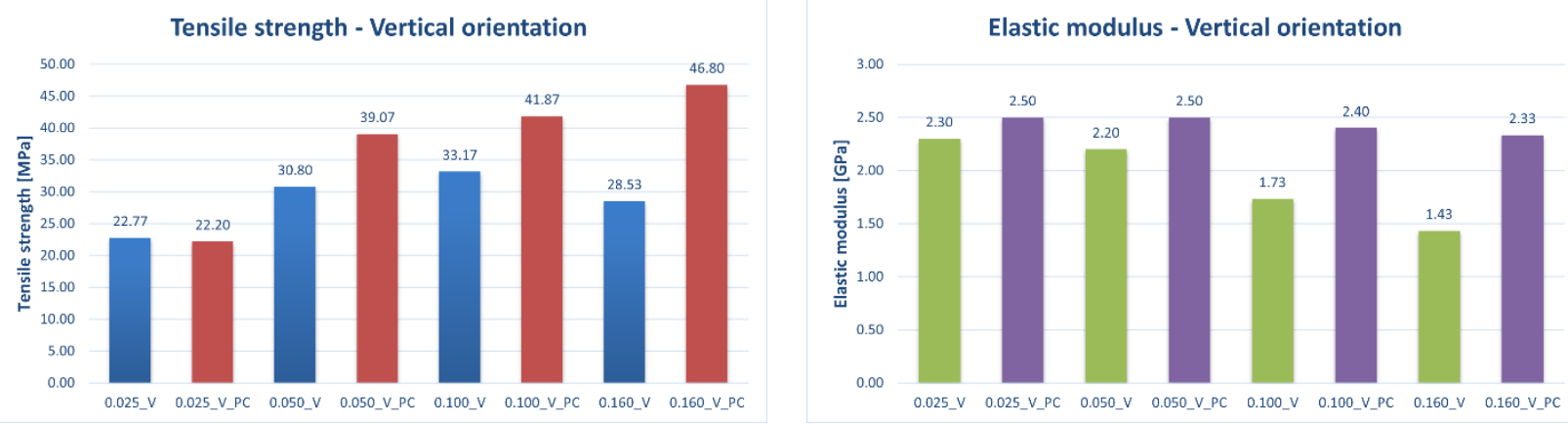

Fig. 12. Influence of $\mathrm{LH}$ and PC on tensile strength and elastic modulus for specimens printed in vertical BO

Influence of build orientation on tensile strength and elastic modulus is presented on diagrams below (Fig. 13). As expected, and based on these diagrams, it can be concluded, that the build orientation during SLA printing has an impact on the tensile mechanical properties. Results also showing that depending on the layer height, the impact of the build orientation changes, and specimens with lower layer height showed higher impact of build orientation on tensile strength. Analysing all samples, with and without post curing, for samples SLA printed in flat and side build orientation, the difference in tensile strength does not exceed 6\%. For elastic modulus, in general, vertical build orientation is showing highest elastic modulus as expected.

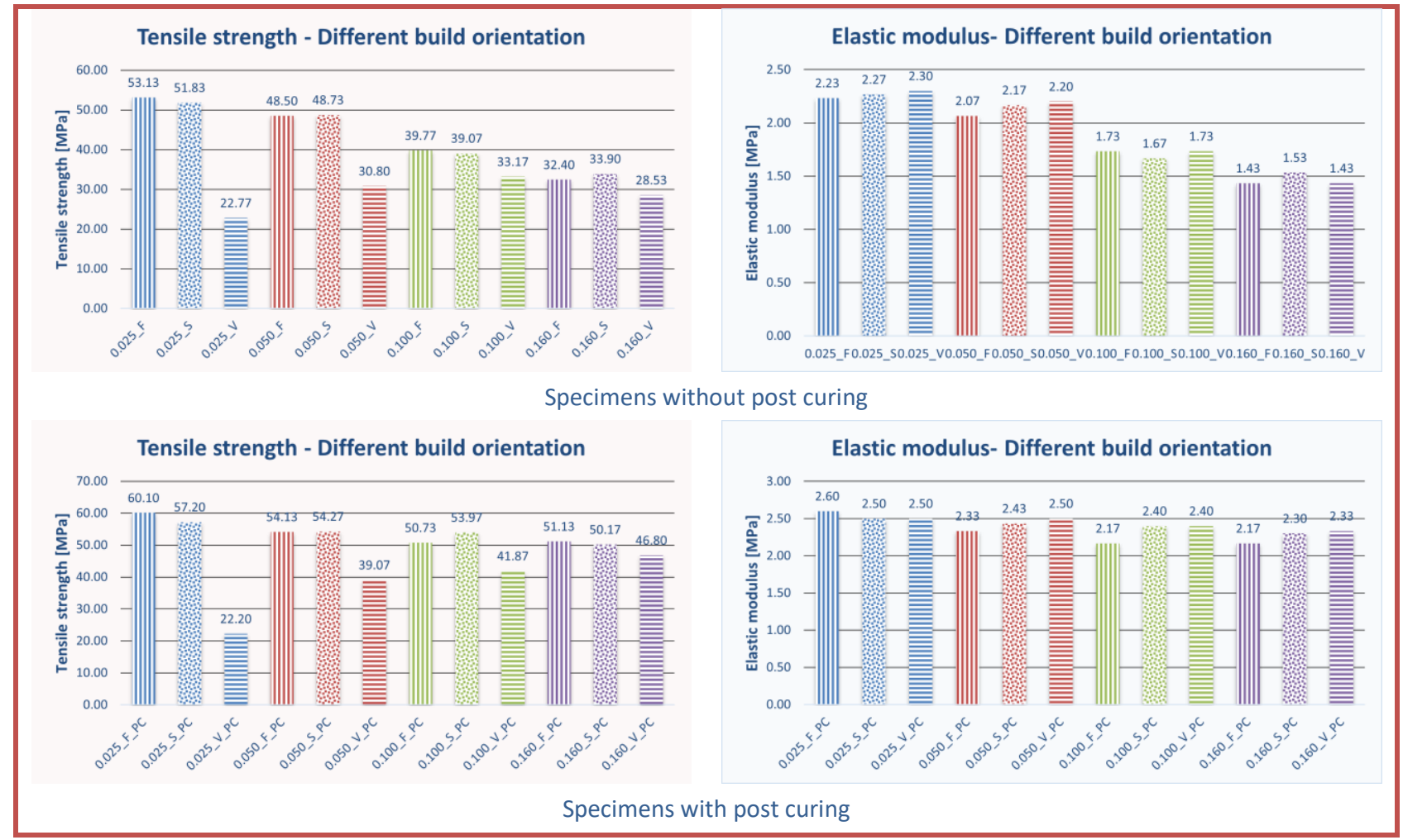

Fig. 13. Influence of build orientation on tensile strength and elastic modulus for tested specimens with and without post curing

It is known that the layer height and build orientation affect the time of product manufacturing by 3D printing, which can be seen from diagrams in Fig. 14. As expected, when the layer height decreases, the printing time increases. Analysing build orientation, the maximum printing time is achieved with vertical, then with the side and flat build orientation. Vertical build orientation increases printing time up to 6 times compared to side and flat build orientation. Post cure generally increased tensile strength and modulus of elasticity, which could be inferred from previous results as well.

When printing time is analysed, which is important in most cases because it directly influence on final product cost, from diagrams it can be seen that with post curing we can print products with higher layer height and achieved mechanical properties similar or higher than specimens with lower layer height, and with that, save the printing time and final product cost. In this research, post curing was 30 min which are added on overall manufacturing time (printing time + post curing time) and presented in diagrams (Fig. 14). So based on the results from diagrams, it can be concluded that with post curing after SLA printing, mechanical properties can be achieved with saving overall manufacturing time. 


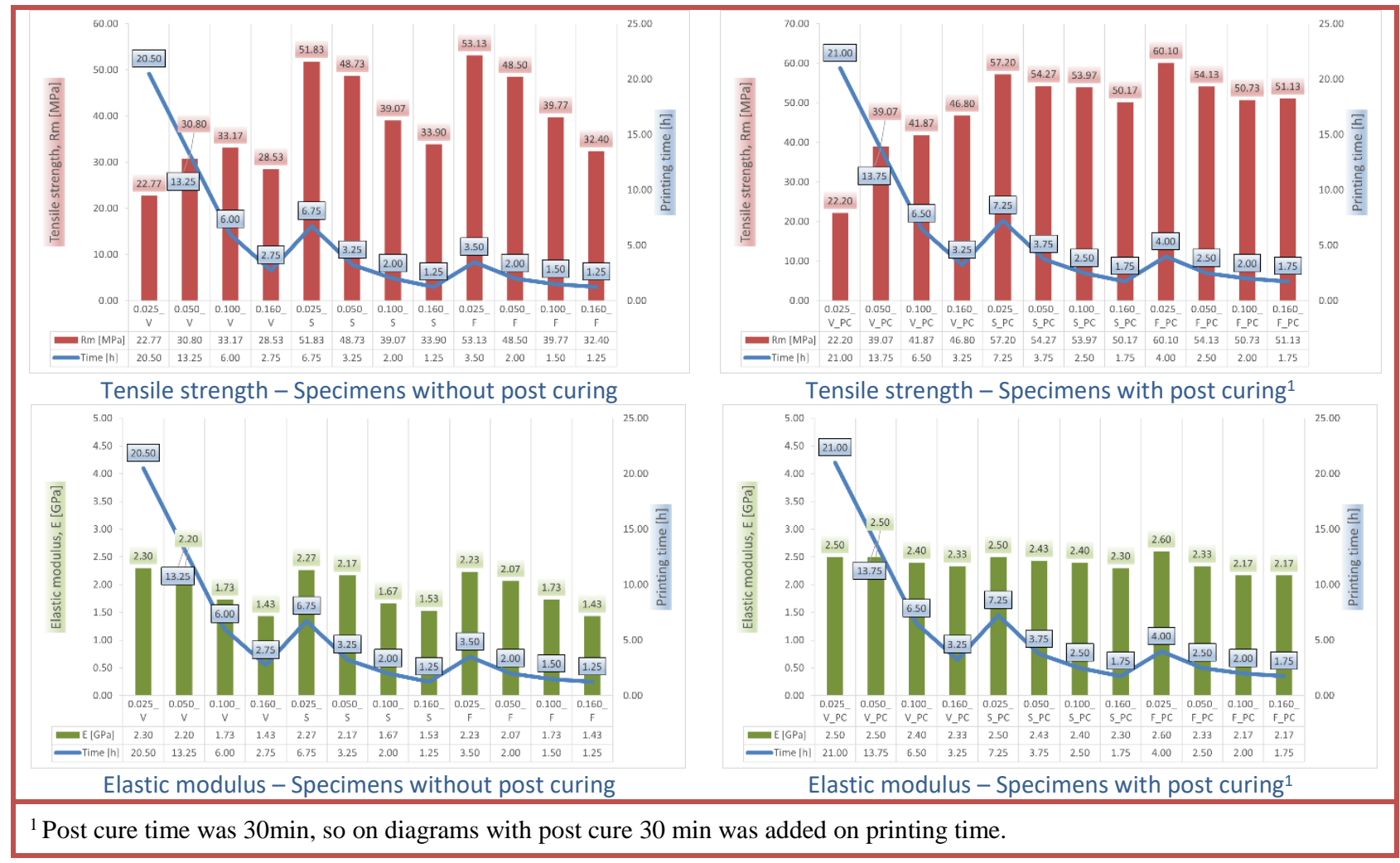

Fig. 14. Influence of layer height, build orientation and post cure on printing time for tested specimens with and without post curing

\section{Conclusion}

Through this experimental research in this study, the influence of layer height, build orientation and post cure on tensile mechanical properties of "Grey resin" (by Formlabs) material SLA 3D printed on a Form 3B device was analysed. The results showed the importance of all three parameters for manufacturing SLA products (in this work tensile specimens) with the aim of obtaining the appropriate tensile strength at an acceptable cost. Three replicas were printed for each combination of parameters, so that a total of 72 tensile specimens were examined according to ISO 527-2, and with strain rate of $5 \mathrm{~mm} / \mathrm{min}$. Based on the results from this study, the following conclusions were drawn:

- Layer height have influence on tensile strength and elastic modulus. For samples SLA printed in flat and side build orientations, a linear increase in tensile strength and elastic modulus was shown with a decrease in layer height. While for samples printed in vertical build orientation, a lower layer height showed lower tensile strength.

- Build orientation also affects the tensile strength and elastic modulus, but the intensity of the impact depends on the layer height. Vertical build orientation increases printing time up to 6 times compared to side and flat build orientation. What can also be concluded is that flat and side build orientation show small differences in tensile strength and elastic modulus. For layer height of 160 microns difference in tensile strength for specimens without post curing is less than $17 \%$, and after post curing the difference is less than $9 \%$.

- Post curing according to the manufacturer's recommendations for this "Grey resin" material, $30 \mathrm{~min}$ at $60{ }^{\circ} \mathrm{C}$, really had the effect of increasing the tensile mechanical properties, where the increase in tensile strength went up to $48 \%$ and in elastic modulus up to $47 \%$, depending on layer height and build orientation. Also, post curing reduced the difference of tensile strength and elastic modulus for samples made with different layer height.

- Analysing printing time, it can be concluded that with a decrease in layer height, the production time increases, as well as with a different build orientation (increases from flat, side to vertical).

In addition to the above conclusions, if the goal is to reduce the printing time and to achieve adequate strength, this can be done using post curing after printing, so for future research it would be good to do optimization of these parameters. It is also recommended to analyse the influence of different post curing parameters (curing time and temperature), and to examined similar experiment but for other material mechanical properties such as compressive, flexural, etc. And detailed analysis of the impact of various factors and printing parameters on other SLA materials (resins) is of great importance in order to be able to make the most of the advantages of this technology.

\section{Acknowledgments}

The author would like to acknowledge the "3D Republika" company for supporting this research. 


\section{References}

[1] Pandzic, A.; Hodzic, D.; Hajro, I. \& Tasic, P. (2020). Strength Properties of PLA Material Obtained by Different Models of FDM 3D Printer, Proceedings of the 31st DAAAM International Symposium on Intelligent Manufacturing and Automation, Mostar, ISSN: 1726-9679, ISBN 978-3-902734-29-7, Katalinic, B. (Ed.), pp. 03150322, Published by DAAAM International, Vienna, DOI: 10.2507/31st.daaam.proceedings.044

[2] Cekic, A.; Begic-Hajdarevic, Dz.; Cohodar, M.; Muhamedagic, K. \& Osmanlic, M. (2019). Optimization of Stereolitography and Fused Deposition Modeling Process Parameters, Proceedings of the 30th DAAAM International Symposium on Intelligent Manufacturing and Automation, Zadar, ISSN: 1726-9679, ISBN 978-3902734-22-8, Katalinic, B. (Ed.), pp. 0681-0687, Published by DAAAM International, Vienna, DOI: 10.2507/30th.daaam.proceedings.093

[3] Pandzic, A.; Hodzic, D. \& Milovanovic, A. (2019). Effect of Infill Type and Density on Tensile Properties of PLA Material for FDM Process, Proceedings of the 30th DAAAM International Symposium on Intelligent Manufacturing and Automation, Mostar, ISSN: 1726-9679, ISBN 978-3-902734-22-8, Katalinic, B. (Ed.), pp. 0545-0554, Published by DAAAM International, Vienna, DOI: 10.2507/30th.daaam.proceedings.074

[4] Castro-Sastre, A.; Fernandez-Abia, A. I.; Rodriguez-Gonzalez, P.; Martinez-Pellitero, S. \& Barreiro, J. (2018). Characterization of materials Used in 3D-Printing Technology With Different Analysis Techniques, Proceedings of the 29th DAAAM International Symposium on Intelligent Manufacturing and Automation, Zadar, ISSN: 1726-9679, ISBN 978-3-902734-20-4, Katalinic, B. (Ed.), pp. 0947-0954, Published by DAAAM International, Vienna, DOI: 10.2507/29th.daaam.proceedings. 136

[5] Pandzic, A.; Hodzic, D. \& Kadric, E. (2021). Experimental Investigation on Influence of Infill Density on Tensile Mechanical Properties of Different FDM 3D Printed Materials, TEM Journal, Vol. 10, No. 3, 2021, pp. 1195-1201, ISSN 2217-8309, DOI: 10.18421/TEM103-25

[6] Dana, M.; Zetkova, I.; Hanzl, P. \& Hronek, J. (2017). Accuracy of Holes Created by 3D Printing (DMLS), Proceedings of the 28th DAAAM International Symposium on Intelligent Manufacturing and Automation, Zadar, ISSN: 1726-9679, ISBN 978-3-902734-11-2, Katalinic, B. (Ed.), pp. 0467-0473, Published by DAAAM International, Vienna, DOI: 10.2507/28th.daaam.proceedings.065

[7] Walcawik-Macura, E. \& Krol, M. (2021). Influence of Post-Processing and the Type of Filling on Strength Properties of Elements Printed by Stereolithography Technology, Solid State Phenomena, Vol. 236, pp. 51-60, Trans Tech Publications Ltd, Switzerland

[8] Yun, J. S.; Tae-Wan, P.; Jeong, Y. H. \& Cho, J. H. (2016). Development of Ceramic-Reinforced Photopolymers for SLA 3D Printing Technology, Applied Physics A 122, 629, DOI: 10.1007/s00339-016-0157-x

[9] Dizon, J.R.C.; Gache, C.C.L.; Cascolan, H.M.S.; Cancino, L.T. \& Advincula, R.C. (2021). Post-Processing of 3DPrinted Polymers, Technologies, Vol. 9, No. 3, DOI: 10.3390/technologies9030061

[10] Saini, J.S.; Dowling, L.; Kennedy, J. \& Trimble, D. (2020). Investigations of the Mechanical Properties on Different Print Orientations in SLA 3D Printed Resin, Proceedings of the Institution of Mechanical Engineers, Part C: Journal of Mechanical Engineering Science, Vol. 234, No. 11, pp. 2278-2293, DOI: 10.1177/0954406220904106

[11] Xiaoyan, X.; Goyanes, A.; Trenfield, S.J.; Diaz-Gomez, L.; Alvarez-Lorenzo, C.; Gaisford, S. \& Basit, A. W. (2020). Stereolithography (SLA) 3D Printing of a Bladder Device for Intravesical Drug Delivery, Materials Science \& Engineering C, Vol. 120, 10.1016/j.msec.2020.111773

[12] Cosmi, F. \& Dal Maso, A. (2020). A Mechanical Characterization of SLA 3D-Printed Specimens for Low-Budget Applications, Materials Today: Proceedings, Vol. 32, No. 2, pp. 194-201, DOI: 10.1016/j.matpr.2020.04.602

[13] Taneva, I. \& Uzunov, T. (2020). Influence of Post-Polymerization Processing on the Mechanical Characteristics of 3D-Printed Occlusal Splints, Journal of Physics: Conference Series, 1492, DOI: 10.1088/1742-6596/1492/1/012018

[14] Espino, M. T.; Tuazon, B. J.; Robles, G. S. \& Dizon, J. R. C. (2020). Application of Taguchi Methodology in Evaluating the Rockwell Hardness of SLA 3D Printed Polymers, Materials Science Forum, Vol. 1005, pp. 166-173, DOI: 10.4028/www.scientific.net/MSF.1005.166

[15] Dall-Argine, C.; Hochwallner, A.; Klikovits, N.; Liska, R.; Stampf, J. \& Sangermano, M. (2020). Hot-Lithography SLA-3D Printing of Epoxy Resin, Macromolecular Materials and Engineering, Vol. 305, No. 10, DOI: 10.1002/mame.202000325

[16] Miedzinska, D.; Gieleta, R. \& Ma-Lek, E. (2019). Experimental Study of Strength Properties of SLA Resins Under Low and High Strain Rates, Mechanics of Materials, Vol. 141, DOI: 10.1016/j.mechmat.2019.103245

[17] Shim, J. S.; Kim, J. E.; Jeong, S. H.; Choi, Y. J. \& Ryu, J. J. (2020). Printing Accuracy, Mechanical Properties, Surface Characteristics, and Microbial Adhesion of 3D-Printed Resins With Various Printing Orientation, The Journal of Prosthetic Dentistry, Vol. 124, No. 4, pp. 468-475, DOI: 10.1016/j.prosdent.2019.05.034

[18] Bryant, S. J. \& Mcleod, R. R. (2018). Understanding and Improving Mechanical Properties in 3D Printed Parts Using a Dual-Cure Acrylate-Based Resin for Stereolithography, Advanced Engineering Materials, Vol. 20, No. 12, DOI: $10.1002 /$ adem.201800876

[19] Watters, M. P. \& Bernhardt, M. L. (2018). Curing Parameters to Improve the Mechanical Properties of Stereolithographic Printed Specimens, Rapid Prototyping Journal, Vol. 24, No. 1, DOI: 10.1108/RPJ-11-2016-0180 